ACCEPTED MANUSCRIPT

\title{
Surface profile and topography filtering by Legendre polynomials
}

To cite this article before publication: Han Haitjema 2021 Surf. Topogr.: Metrol. Prop. in press https://doi.org/10.1088/2051-672X/abe214

\section{Manuscript version: Accepted Manuscript}

Accepted Manuscript is "the version of the article accepted for publication including all changes made as a result of the peer review process, and which may also include the addition to the article by IOP Publishing of a header, an article ID, a cover sheet and/or an 'Accepted

Manuscript' watermark, but excluding any other editing, typesetting or other changes made by IOP Publishing and/or its licensors"

This Accepted Manuscript is @ 2021 IOP Publishing Ltd.

During the embargo period (the 12 month period from the publication of the Version of Record of this article), the Accepted Manuscript is fully protected by copyright and cannot be reused or reposted elsewhere.

As the Version of Record of this article is going to be / has been published on a subscription basis, this Accepted Manuscript is available for reuse under a CC BY-NC-ND 3.0 licence after the 12 month embargo period.

After the embargo period, everyone is permitted to use copy and redistribute this article for non-commercial purposes only, provided that they adhere to all the terms of the licence https://creativecommons.org/licences/by-nc-nd/3.0

Although reasonable endeavours have been taken to obtain all necessary permissions from third parties to include their copyrighted content within this article, their full citation and copyright line may not be present in this Accepted Manuscript version. Before using any content from this article, please refer to the Version of Record on IOPscience once published for full citation and copyright details, as permissions will likely be required. All third party content is fully copyright protected, unless specifically stated otherwise in the figure caption in the Version of Record.

View the article online for updates and enhancements. 


\title{
Surface profile and topography filtering by Legendre polynomials
}

\author{
Han Haitjema ${ }^{1}$ \\ E-mail: han.haitjema@kuleuven.be \\ Received xxxxxx \\ Accepted for publication $\mathrm{xxxxxx}$ \\ Published xxxxxx
}

${ }^{1}$ Manufacturing Metrology Section, Manufacturing Processes and Systems (MaPS), Mechanical Engineering department, KU Leuven, Leuven, Belgium

\section{Abstract}

A surface filtering method based on Legendre polynomials is proposed that can be used to separate form and roughness of surface profiles and areal topographies. The filter has been designed in such a way that it well approaches the properties of a Gaussian filter, especially in the center of the measured region. Theoretical profiles and measurement examples show that there is little difference to the zero- and second order Gaussian regression filters, both in the obtained topography and in the calculated profile- and areal parameters. Compared to the Gaussian regression filters the filtering becomes gradually weaker outside the center of the profile/area, while for the Gaussian Regression filters the filtering is constant over the profile/area, but becomes weaker at the edge region within half of the L-nesting index distance. Especially for cases where the nesting index is equal to or not much smaller than the dimensions of the measured area, such as the default case according to ISO 25178-2:2012, the principal advantage of the polynomial filter is that the form removal is an integral part of the filtering, so it does not interact with the filter and does not need to be carried out separately. Also the reference plane is principally unchanged after application of this filter. Some applications are shown and a fast numerical implementation is given.

Keywords: Legendre polynomial, filter, form, roughness

\section{Introduction}

The separation between form and roughness is a classical issue in surface topography measurements. Historically, contact stylus instruments that provided a monotonous speed over a surface were equipped with a double high-pass CR filter that removed the form from the profile. In the 1990's digital processing of the texture became common, and the Gaussian filter became the default filter [1]. However still some problems remained such as the discontinuity at the end of the measured profile. Most instruments solve this issue by measuring a run-in and run-out part of typically half a cut-off length; and Gaussian regression filters were defined that adapt the weighing function at the beginning and the end of a profile [2]. The form/roughness separation becomes a critical issue if the measurement length is not taken as several cut-off lengths, but is taken as just a single or a few cut-off lengths because of limitations of space and/or bandwidth. In areal surface topography measurements, the measurement range related to the lateral resolution makes it rather unfeasible to measure several cut-off wavelength while keeping a reasonable bandwidth; and probably because of this reason ISO 25178-3:2012 (section 4.2.1.3.) [3] defines 
"For an S-L surface, if not otherwise specified, the evaluation area shall be a square whose sides are the same length as the L-filter nesting index value.". In addition section 4.4.3 specifies: "The default L-filter is an areal Gaussian filter (see ISO 16610-21)".

When a single cut-off length (L-nesting index) is taken as the measurement length, the filter operation may interfere with the F-(form) operator that removes the form before the L-filter is applied. As normally surface areas are measured without reference to a datum, the form must be removed first normally the least-squares plane is removed - before a filter operation is performed. In addition, for a single L-nesting index the filtering is rather weak: theoretically a cosine with a wavelength equal to the measured area is reduced 50\% and shorter wavelengths are reduced even less; the filtering of longer wavelengths is in fact poorly defined. A single sine with a wavelength equal to the measured area is deformed when first a least-squares line is removed; as was already noted by Whitehouse [1], which illustrates the mixing of form and roughness filtering which makes a proper filtering tedious.

Rather than in Fourier-terms, a surface can as well be described using Legendre polynomials. These polynomials are orthogonal and exhibit some advantages when describing surfaces because the least-squares line (surface) is part of the analysis. This means that the least-squares line removal does not need to be performed as an additional operation that makes the Fourier-terms correlated, as was shown by Haitjema [4]. In this paper a filtering method is proposed based on Legendre polynomials where the Gaussian filter is approximated as well as possible, and where the F-operation is a natural part of the filter operation, avoiding interaction between the F- and L- filtering operations.

\section{Legendre filter design and properties}

\subsection{Filter design}

The Gaussian filter was developed as a phase-correct alternative for the 2CR filter, with a steeper suppression of higher frequencies than the cut-off frequencies, and with a Gaussian characteristics in both the time- and frequency domain. Also it was designed for continuity: on 'average' the surface parameters should not change when using the $2 \mathrm{CR}$ or the Gaussian filter, just because of the phase-correctness it no longer mattered whether a surface was probed from the left to the right or from the right to the left. Still two issues remained: edge effects, and a form removal needed prior to the filtering.

To maintain a similar continuity, a similar approach as was used for defining a Gaussian filter according to ISO 16610-21:2011 [5] is taken, however because Legendre polynomials are defined on a limited interval and, contrary to Fourier coefficients, no periodicity needs to be assumed, edge effects are expected to need no special precautions, and the form removal can be an integral part of the filter. Therefore the basis of this filter is the weighting function of the Gaussian filter. The weighting function of the Gaussian filter is given by:

$$
s(x)=\frac{1}{\alpha \cdot \lambda_{c}} \exp \left(-\pi\left(\frac{x}{\alpha \cdot \lambda_{c}}\right)^{2}\right)
$$

where $x$ is the distance from the center (maximum) of the weighting function, $\lambda_{c}$ is the cut-off wavelength and $\alpha$ is a constant, $\alpha=\sqrt{\log (2) / \pi}$, to provide a $50 \%$ transmission characteristic at the cutoff wavelength $\lambda_{c}$. The filter mean line $w(x)$ emerges from the convolution of the measured profile $z(x)$ with the weighting function $s(x)$ :

$$
w(x)=\int_{-\infty}^{\infty} z(x-\xi) \cdot s(\xi) d \xi
$$

This is equivalent to a weighted averaging of the profile $z(x)$. When taking the delta-function for $z(x): z(x)=\delta(x), w(x)$ is given by $s(x)$. In the frequency domain, the frequency characteristic is given by 


$$
S(f)=\frac{F T(s(x))}{F T(\delta(x))}
$$

Where $F T$ is the Fourier transform and $f$ is the spatial frequency. This principle is taken as a basis for defining a filtering operation based on Legendre polynomials. If the $x$-axis is re-scaled to the interval $[-1 ; 1]$, the profile $z(x)$ can be written as:

$$
z(x)=\sum_{k=0}^{\infty} \hat{z}(k) \cdot P(x, k)
$$

where $\hat{z}(k)$ is the Legendre transform of $z(x)$ and $P(x, k)$ is the Legendre polynomial of degree $k$. Analogous to (3) a polynomial filter characteristic $S_{L}(k)$ can be defined as:

$$
S_{L}(k)=\frac{L T(s(x))}{L T(\delta(x))}
$$

Where $L T$ is the Legendre transform. From this, the Legendre-filtered mean line $w_{L}(x)$ is given as

$$
w_{L}(x)=\sum_{k=0}^{\infty} S_{L}(k) \cdot \hat{z}(k) \cdot P(x, k),
$$

and the 'high-pass' Legendre-filtered profile $z_{L}(x)$ is given by:

$$
z_{L}(x)=\sum_{k=0}^{\infty}\left(1-S_{L}(k)\right) \cdot \hat{z}(k) \cdot P(x, k)=z(x)-w_{L}(x)=z(x)-\sum_{k=0}^{\infty} S_{L}(k) \cdot \hat{z}(k) \cdot P(x, k) .
$$

In practice a profile will be available as discrete coordinates in digital form: $z(x)$ with $x=0 . . N$. For this case the discrete Legendre transform $(D L T)$ can be written as a matrix operation:

with [6]:

$$
\operatorname{DLT}(z(x))=C(k)=\boldsymbol{M}_{\boldsymbol{k}, \boldsymbol{x}} \cdot z(x)
$$

$$
\boldsymbol{M}_{\boldsymbol{k}, \boldsymbol{x}}=p_{n}(x, k, N)=\sum_{l=0}^{k}(-1)^{l}\left(\begin{array}{c}
k \\
l
\end{array}\right)\left(\begin{array}{c}
k+l \\
l
\end{array}\right) \frac{N^{(k)}(2 k+1) x^{(l)}}{(N+k+1)^{(k+1)} N^{(l)}}
$$

where $p_{n}$ is the normalized discrete Legendre polynomial, $x^{(l)}$ is the backward factorial function defined as a product of $l$ terms: $x(l)=x \cdot(x-1) \cdot \ldots \cdot(x-l+1)$ and $\left(\begin{array}{l}k \\ l\end{array}\right)$ is the binomial coefficient defined by $\left(\begin{array}{l}k \\ l\end{array}\right)=\frac{k !}{l !(k-l) !}$.

The polynomial filter characteristic $S_{L}(k)$ for a discretized profile can be constructed as:

$$
S_{L}(k)=\frac{D L T(s(x-N / 2))}{D L T(\delta(x-N / 2))}
$$

where $\lambda_{c}$ in (1) is taken as the number of points that correspond to $\lambda_{c}$. This defines a filter that transforms a $\delta$-function in the center of the profile to the Gaussian characteristic as in (1). The mean line follows from

$$
w_{L}(x)=\boldsymbol{M}^{\prime} \cdot\left(S_{L}(k) \cdot C(k)\right)
$$

As an illustration the filter characteristics $S_{L}(k)$ as calculated from (10) is given for $N=2000$ and $\lambda_{c}=500$ in figure 1 . 


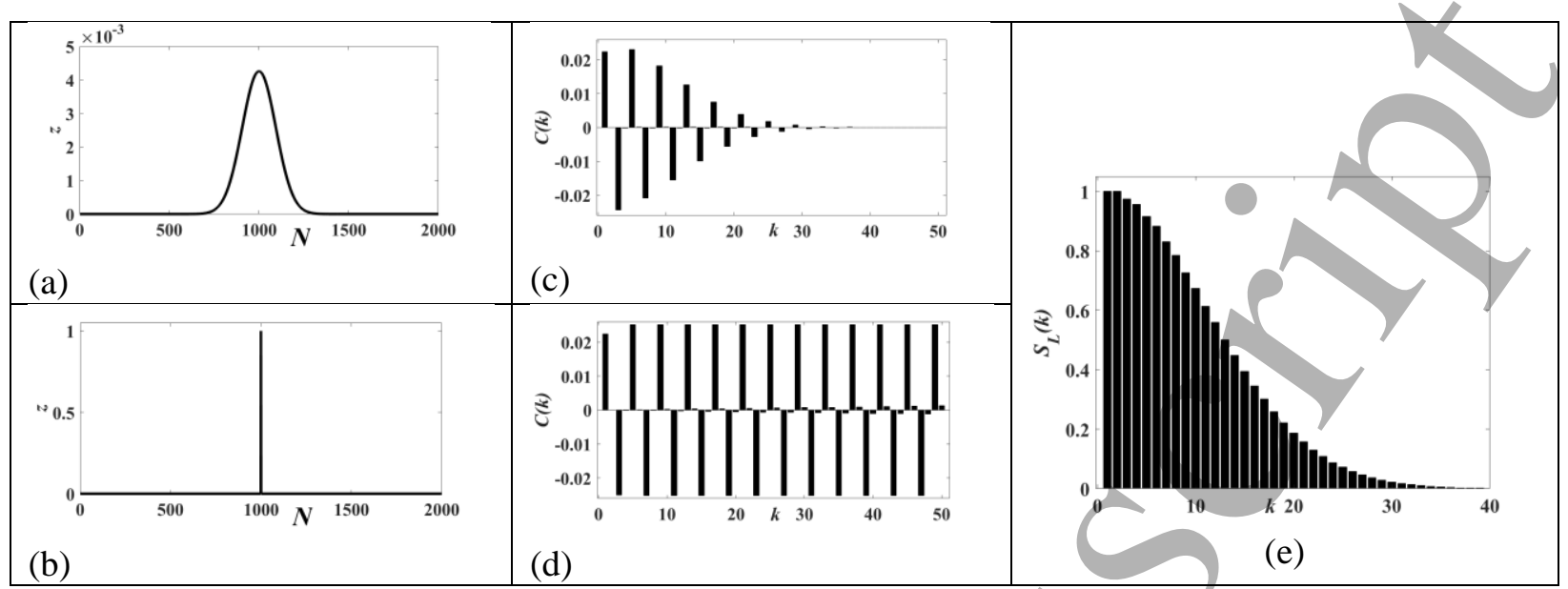

Figure 1. Graphical illustration of the calculation of the filter function $S_{L}(k)$ (figure e) from the Legendre transform (figure $\mathrm{c}$ and d) of the Gaussian filter characteristics (figure a) and the delta-function (b) respectively.

Figure 1 illustrates the calculation of $S(k)$ from the discrete Legendre transforms of the delta-function and the Gaussian filter characteristics as given in (10). Figure 1(e) shows a Gaussian form of the Legendre filter function $S_{L}(k)$ as well that can be approximated by

$$
S_{L a}(k)=\exp \left(-\log (2)\left(\frac{k}{k_{c}}\right)^{2}\right),
$$

With $k_{c}=12$ the cut-off polynomial degree for which $S_{L a}(k)=0.5$. A similar Gaussian form of the function $S_{L}(k)$ was found for other values of $\lambda_{c}$ as well. The value of $k_{c}$ appeared to vary linearly with $L / \lambda_{c}$, where $L$ is the profile length, as is shown in Figure 2.

Using the coefficients of the linear least-squares fit of $k_{c}$ to $L / \lambda_{c}$ it was found that for $1 \leq L / \lambda_{c} \leq 7$ the filter function $S_{L}(k)$ can well be approximated by:

$$
S_{L a}(k)=\exp \left(-\left(\frac{k}{3.8 \cdot L / \lambda_{c}-0.76}\right)^{2}\right) \text {, }
$$

In addition, $S_{L a}(1)=1$ should be defined in order to fully remove the least-squares line. For $L / \lambda_{c}<1$ the filtering effect is very small and filtering is not considered useful. For $L / \lambda_{c}>7$ polynomial orders $k>50$ must be calculated that cannot be done numerically stable; so this Legendre filter is limited to a high-pass/low pass filter as long as polynomial coefficients $k \leq 50$ are involved and higher orders are either completely filtered or remain unchanged. As an illustration the function $S_{L a}$ calculated from (13) and $S_{L}$ calculated from (10) are given for $L / \lambda_{c}=1$ (this corresponds to the default case according to ISO 25178-3:2012 [3] ), $L / \lambda_{c}=3 / 2$ and $L / \lambda_{c}=2$ and $N=2000$ in figure 3 . 


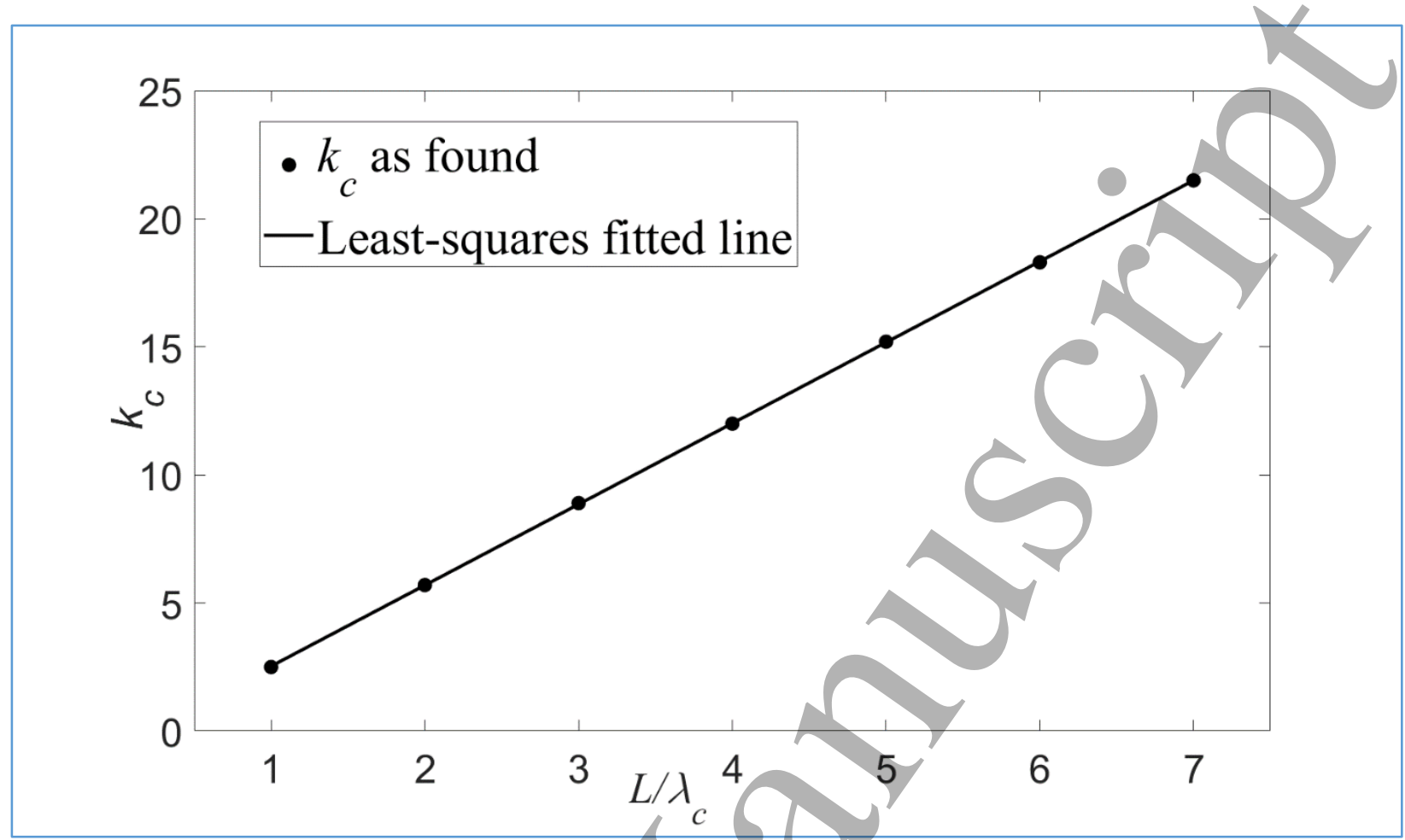

Figure 2. Dependence of the found value of $k_{c}$ on the $L / \lambda_{c}$ ratio

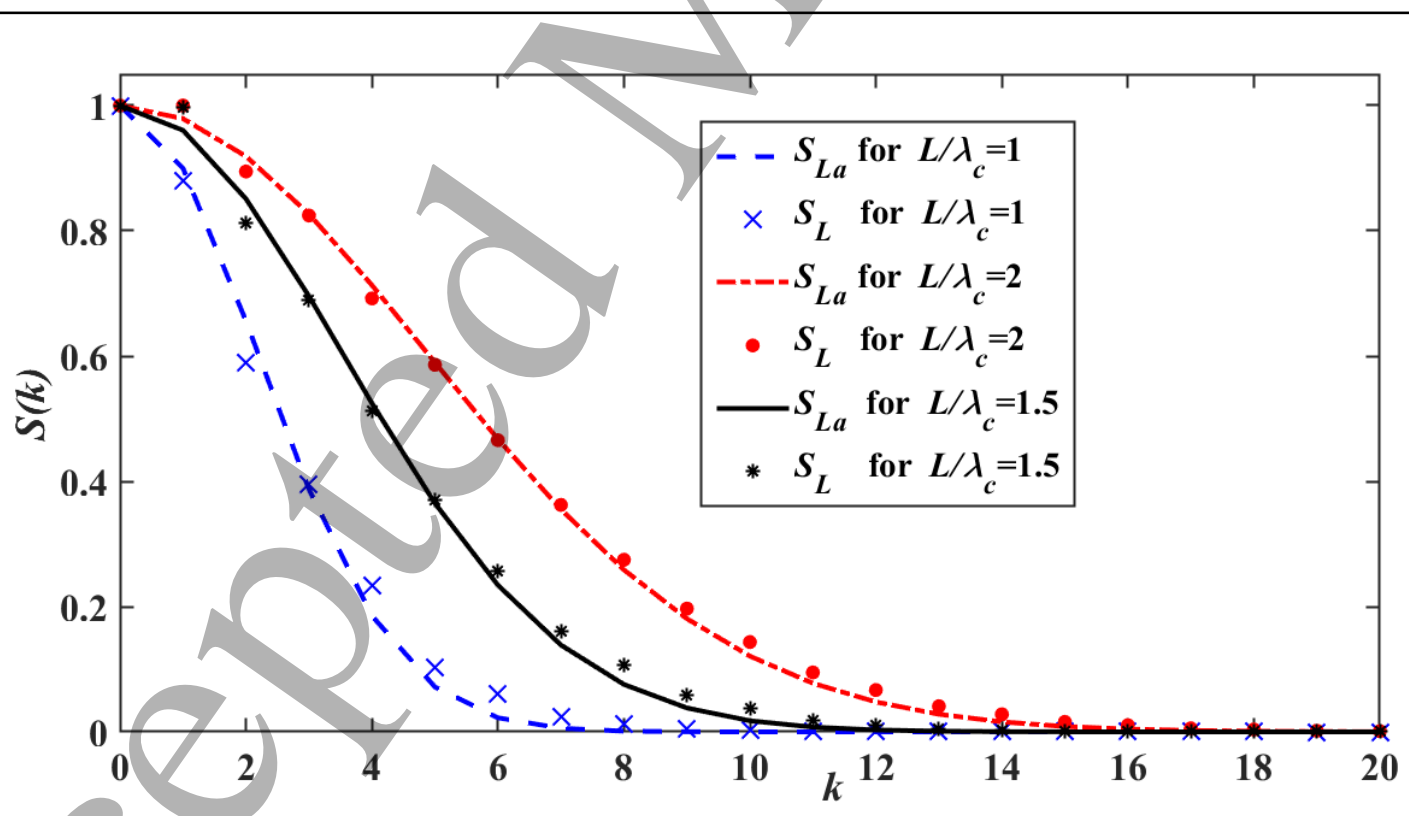

Figure 3. Filter functions $S_{L a}$ and $S_{L}$ for $N=2000$ and $L / \lambda_{c}=1, L / \lambda_{c}=2$ and $L / \lambda_{c}=1.5$

\subsection{Filter properties compared to the Gaussian regression filter}

Unlike the Gaussian filtering, the effect of the Legendre filtering is not uniform over the profile. Near the edges the w-filtering is weaker, that means that the roughness profile is somewhat more filtered. This has some resemblance to the zero-order and second order Gaussian regression filters defined in [2] and [7]. 
The difference with these filters is that the end-effects of the regression filters become effective at the first and last half cut-off length of the profile, while the Legendre filtering becomes gradually weaker towards the edges over the whole profile. This is illustrated in figure 4, where figure 4a gives the filter function $w(x)$ for a zero-order Gaussian regression and figure $4 \mathrm{~b}$ gives $w_{L}(x)$ for the Legendre filter for deltafunctions similar as in figure 1(b) which are now located between $x=100 \mu \mathrm{m}$ and $x=1000 \mu \mathrm{m}$ with steps of $100 \mu \mathrm{m}$; for a profile length $L=2000 \mu \mathrm{m}$ containing 2000 points and a cut-off length $\lambda_{c}=1000 \mu \mathrm{m}$, so $L / \lambda_{c}=2$.

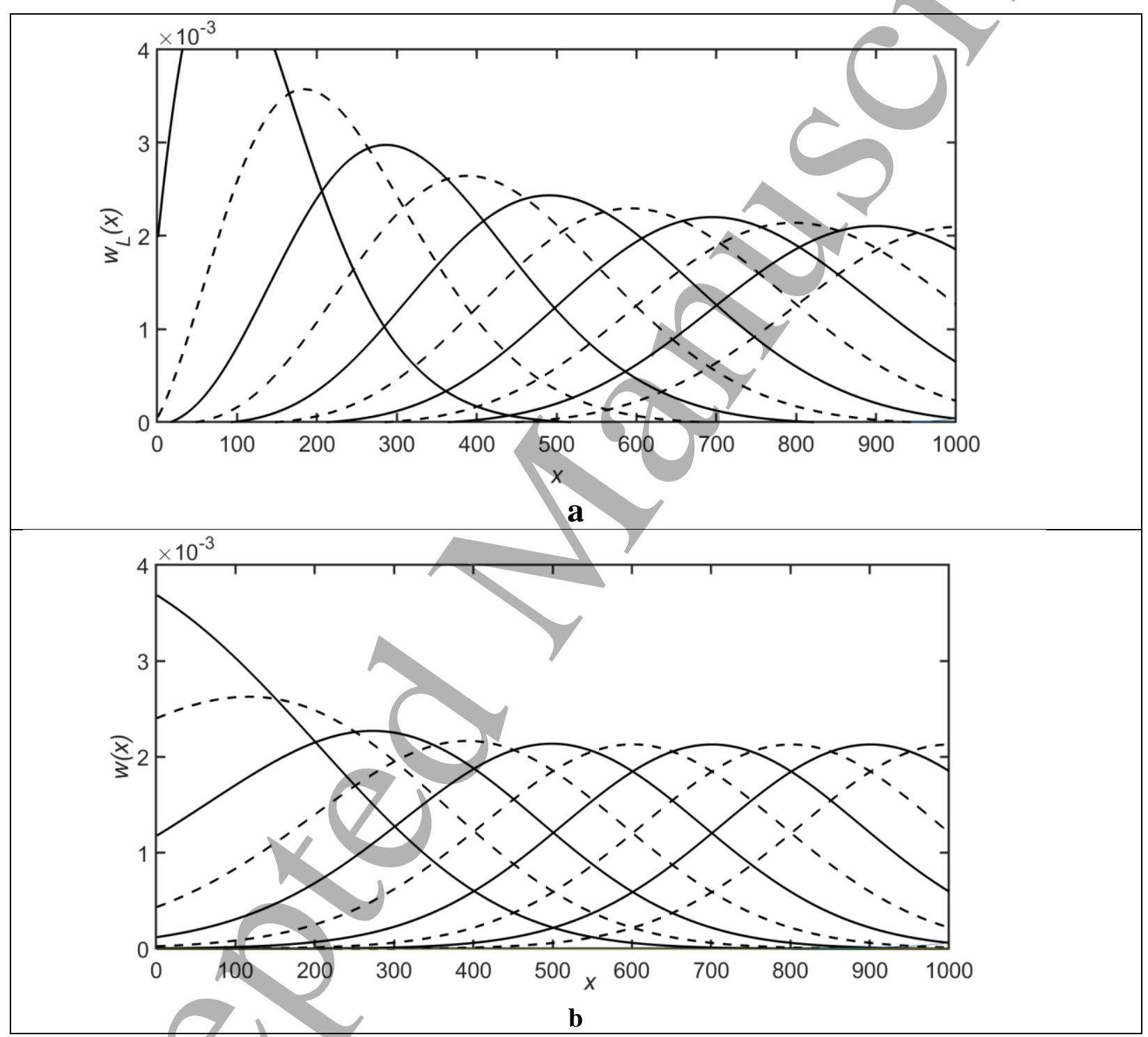

Figure 4. Profiles of $w(x)$ of delta-functions (peaks) at $x=100 \mu \mathrm{m} . . . x=1000 \mu \mathrm{m}$, filtered by a Legendrefilter (a) and by a zero-order Gaussian regression filter (b) for $L=2000 \mu \mathrm{m}$ and $L / \lambda_{c}=2$

Figure 4 shows that the filtering effect is similar in the center of the profile, but at the edges the Legendre filter is stronger and confined to a smaller region, meaning that near the edges the effect on the roughness profile will be smaller than the zero-order Gaussian regression filter, although the zero-order Gaussian filter will have a smaller filtering effect on the roughness profile, compared to the central region, as well. This is further illustrated in figure 5 where a $4 \mathrm{~mm}$ random profile is given. In order to emphasize the low-frequency modulation this random profile is pre-filtered using a Gaussian filter with $\lambda_{s}=60 \mu \mathrm{m}$. 


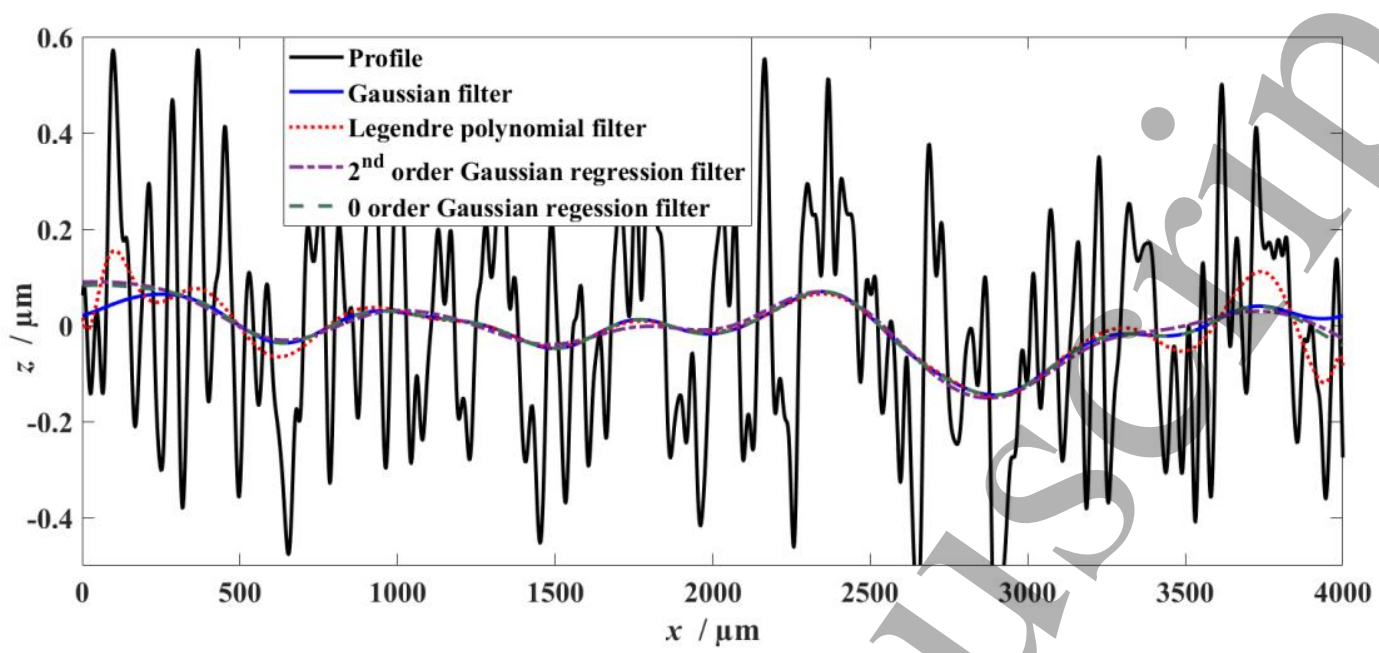

Figure 5. Random profile with the mean line $w(x)$ of different filters applied.

To this profile, a Gaussian filter according to (1) and (2) is applied, as well as the Legendre polynomial filter according to (13) and a zero and second order Gaussian regression filters according to [2] and [7], all with a cut-off length $\lambda_{c}=0.8 \mathrm{~mm}$. The figure shows that in the central region there is little difference between the filters with just a bit deviation of the second order Gaussian regression filter compared to the others, but near the borders the filtering effect of the Legendre polynomial becomes less, even less than the zero and second order Gaussian regression filters. Taking this effect into account, the filter functions $S_{L}(k)$ that best approximate the Gaussian filter (i.e. they keep the same rms $(R q)$ value for a random profile) for $L / \lambda_{c}=1 L / \lambda_{c}=3 / 2$ and $L / \lambda_{c}=2$ were adapted from (13). The resulting filter functions $S_{L}(k)$ are given in Table 1.

Table 1. Legendre filter functions $S_{L}(k)$ for $L / \lambda_{c}=1, L / \lambda_{c}=1.5$ and $L / \lambda_{c}=2$

\begin{tabular}{|c|c|c|c|}
\hline & \multicolumn{3}{|c|}{$S_{L}(k)$} \\
\hline$k$ & $L / \lambda_{c}=1$ & $L / \lambda_{c}=1.5$ & $L / \lambda_{c}=2$ \\
\hline 0 & 1 & 1 & 1 \\
\hline 1 & 1 & 1 & 1 \\
\hline 2 & 0.35 & 0.76 & 0.84 \\
\hline 3 & 0.15 & 0.35 & 0.68 \\
\hline 4 & 0.03 & 0.19 & 0.53 \\
\hline 5 & 0.01 & 0.09 & 0.37 \\
\hline 6 & 0 & 0.04 & 0.23 \\
\hline 7 & 0 & 0.015 & 0.13 \\
\hline 8 & 0 & 0.004 & 0.07 \\
\hline 9 & 0 & 0 & 0.04 \\
\hline 10 & 0 & 0 & 0.02 \\
\hline 11 & 0 & 0 & 0.01 \\
\hline
\end{tabular}

Note that both $S_{L}(0)$ and $S_{L}(1)$ are defined as unity in order to define the removal of the least-squares line, thus incorporating the F-operator in the L-filter definition. The column for $L / \lambda_{c}=1$ also shows that after the removal of the least-squares line there is in fact not much more filtering needed. The resulting profile is aligned according to the least squares line by definition. 
The filter operation can be extended to a 2-D surface topography $z_{L}(x, y)$ consisting of abscissa coordinates $x=0 . . N$ and $y=0 . . M$ as:

$z_{L}(x, y)=z(x, y)-w_{L}(x, y)=z(x, y)-\sum_{l=0}^{M} \sum_{k=0}^{N} S_{L}(k) \cdot C(k) \cdot S_{L}(l) \cdot C(l) \cdot p_{n, k}(x) \cdot p_{n, l}(y)$.

The summations of $k$ and $l$ to $N$ and $M$ respectively in (14) can be limited in practice as $S_{L}(k)$ rapidly reaches zero levels for the smaller $L / \lambda_{c}$ ratios considered in this paper; as illustrated in figure 2 and Table 1. For $L / \lambda_{c}=5$ the filter function $S_{L}(k)$ extends to about $k=40$.

\subsection{Form-filter interaction compared to the Gaussian regression filter}

The absence of a form-filter interaction of the Legendre filter is illustrated by two examples: the first example consists of a comparison using the profile consisting of 2000 points $(x=1.2000 \mu \mathrm{m})$ having a single peak of $z(x)=1$ at $x=100 \mu \mathrm{m}$. In the calculations for figure 4, this profile was not aligned according to the least-squares plane, as would be done if this was a real surface. Filtering the form by removing the least-squares line first affects the filtered profile when the Gaussian filter or the zero-order Gaussian regression filter is used, but the Legendre-filtered roughness profile is not affected by the chosen reference line; and is in fact not affected by the orientation of the profile at all. This is shown in figure 6 that gives - enlarged around $z=0$ - the roughness profile according to (7) when leveled and filtered in different ways. The Gaussian filter obviously suffers from edge-effects which are reduced by the Gaussian regression filter; however the Gaussian (regression) filtered roughness profiles slightly depend on the used reference line, which is not the case with the Legendre filtered profile.

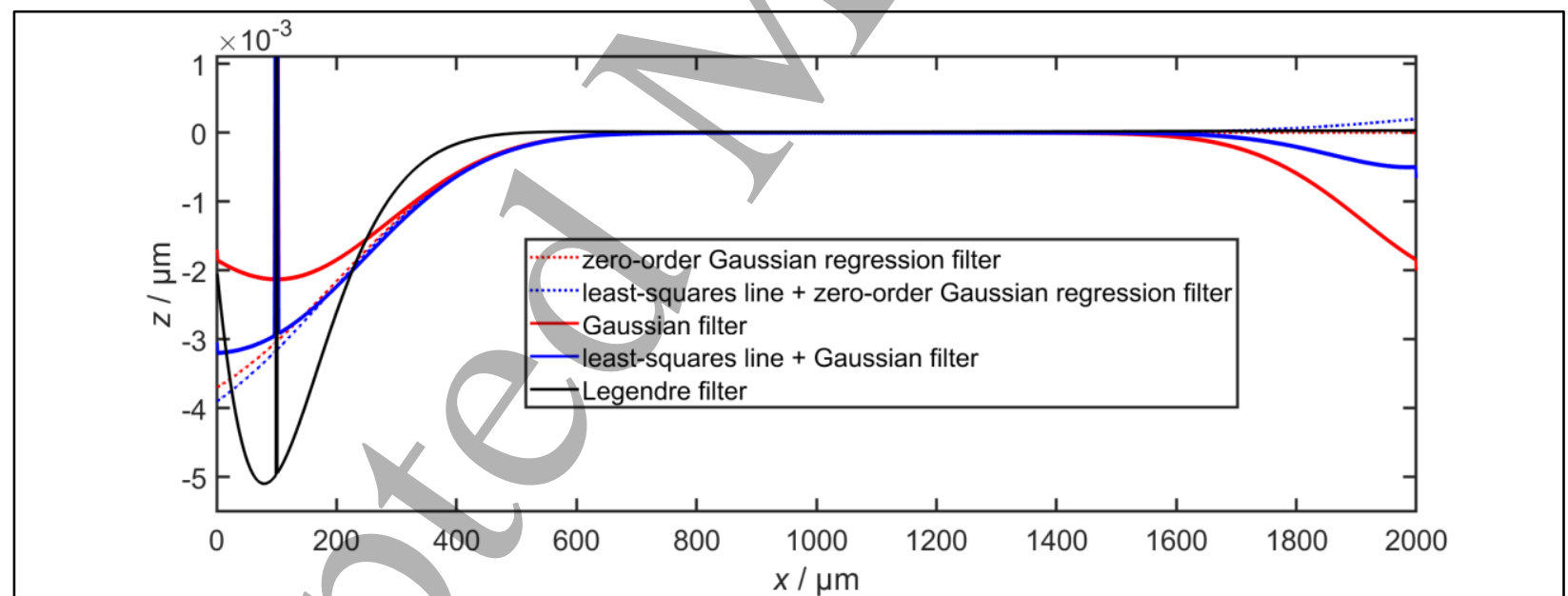

Figure 6. Roughness profiles, enlarged around $z=0$, of a profile containing a delta-function $z(x)=1 \mu \mathrm{m}$ for $x=100 \mu \mathrm{m}$, leveled and filtered in various ways. $\mathrm{z}(100) \approx 1$ for all profiles.

The second example consist of a comparison of a sinusoidal profile with an amplitude of $1 \mu \mathrm{m}$ and a wavelength of $1000 \mu \mathrm{m}$, taken over a $2000 \mu \mathrm{m}$ length. This profile is filtered with $\frac{L}{\lambda_{c}}=2$, so for the Gaussian filter it is expected to be reduced by exactly a factor of two, resulting in a sine wave with an amplitude of $0.5 \mu \mathrm{m}$ as is shown in figure 7 . However when such a profile is measured in practice, it will first be aligned according to the least-squares line before filtering. This affects both the Gaussian filtering and the zero-order Gaussian regression filtering, and results in a distorted filtered profile. For the Legendre polynomial filter the profile is somewhat distorted as well, but it does not depend on the original orientation: the Legendre-filtered roughness profile is identical for both the original as the least- 
squares leveled sine profile. The 'ideal' filtered profile would be the sine-surface with $0.5 \mathrm{x}$ the original amplitude, however aligned according to the least squares line; this line is shown as well in figure 7. Compared to the other filtered profiles, this profile is best approximated by the Legendre filtered profile, especially near the edges.

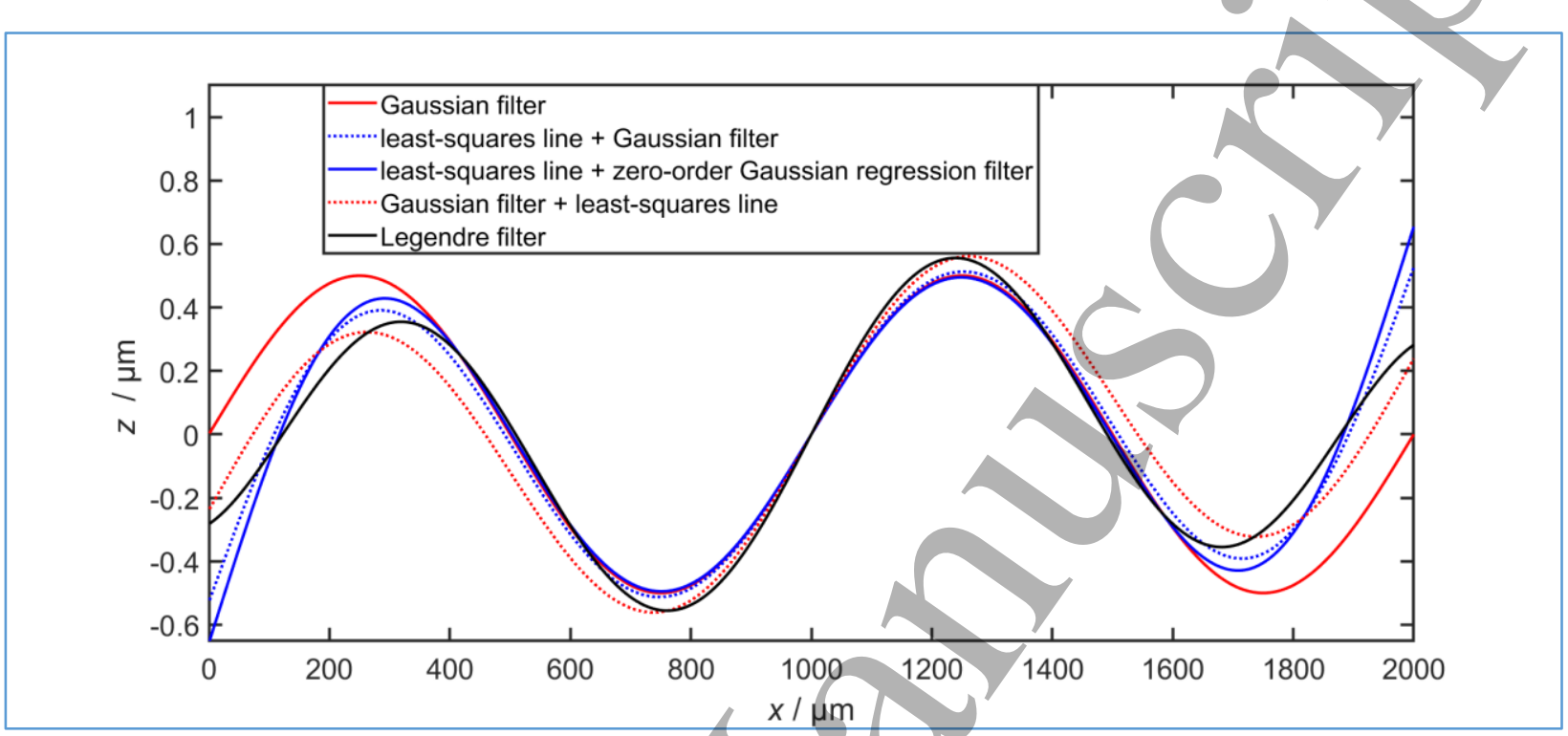

Figure 7. Roughness profile of a sine wave with an amplitude of $1 \mu \mathrm{m}$ and a wavelength of $1000 \mu \mathrm{m}$, filtered by a Gaussian filter, a zero-order Gaussian regression filter and a Legendre polynomial filter.

Other examples can be given using different phases of the sine-signal, or using a few sine waves that do not fit an integer amount of times in the measurement length. In these cases the Legendre filter gives a stable - although never ideal - response where the Gaussian filter and the Gaussian regression filter, either preceded or followed by a least-squares alignment, show varying and sometimes distorted results. This applies when only one or a few sines are present in the profile, for more than 5 sine waves the correlation between the least squares line and the sine signal vanishes and the Gaussian filter gives unambiguous results, while the Legendre polynomials struggle more and more to fit the high-order polynomials.

\section{Measurement example in 1-D}

In this section it is illustrated how the Legendre polynomial filter as defined in section 2 compares to the default Gaussian filter and the Gaussian regression filters as defined in [2] that are the alternatives for small $L / \lambda_{c}$ ratios when edge-effects must be avoided. For this, the profile of a Halle standard roughness specimen, type D1 according to ISO 5436-1 [8] is taken. The measurement was taken using a Mitutoyo CS 3200 formtracer, using a tip radius of nominally $2 \mu \mathrm{m}$ and a tip cone angle of $60^{\circ}$. A trace of $8 \mathrm{~mm}$ was taken using a sampling distance of $0.5 \mu \mathrm{m}$. This profile was leveled according to the least-square line and a Gaussian filter was applied with $\lambda_{c}=0.8 \mathrm{~mm}$. From this profile the central $4 \mathrm{~mm}$ was taken for further evaluation. The reason for this evaluation is that this profile, although random, is repeatable over its measuring length of $4 \mathrm{~mm}$. This enables a well-defined Gaussian filtering in the frequency domain for the central $4 \mathrm{~mm}$ range, as beyond this range the profile is continued periodically and thus it does not have end effects when it is filtered in the frequency domain according to ISO 1660-21 [5].

The 5 separate cut-off lengths $\lambda_{c}=0.8 \mathrm{~mm}$ are used to simulate measurements of a $0.8 \mathrm{~mm}$ range filtered at a single cut-off length. These measurement are filtered in various ways and the filtered profiles and the mean of the derived parameters are compared to the Gaussian filtered $4 \mathrm{~mm}$ profile and the mean parameters calculated from this profile. For comparison each of the $0.8 \mathrm{~mm}$ lengths were processed using: 
- A least squares line (i.e. what is normally done when applying 'no filter')

- The Legendre polynomial filter for $L / \lambda_{c}=1$ as defined in table 1

- A least squares line and the zero-order Gaussian regression filter according to [2]

- A least squares line and the second-order Gaussian regression filter according to [2]

- A second order polynomial

This is what can normally be done if only the $0.8 \mathrm{~mm}$ profile is available. This may be the case in practice when the $4 \mathrm{~mm}$ profile is not or cannot be measured, or when just a piece on $0.8 \mathrm{~mm}$ is cut out of the workpiece, or when a $0.8 \mathrm{~mm} \times 0.8 \mathrm{~mm}$ area of this unidirectional profile is measured that is to be evaluated according to ISO 25178-3 [3].

The profile and the w-filter profiles are shown in figure 8. As the zero- and second order Gaussian regression filter show hardly visible differences, the zero-order Gaussian regression filter profile is omitted in this figure.

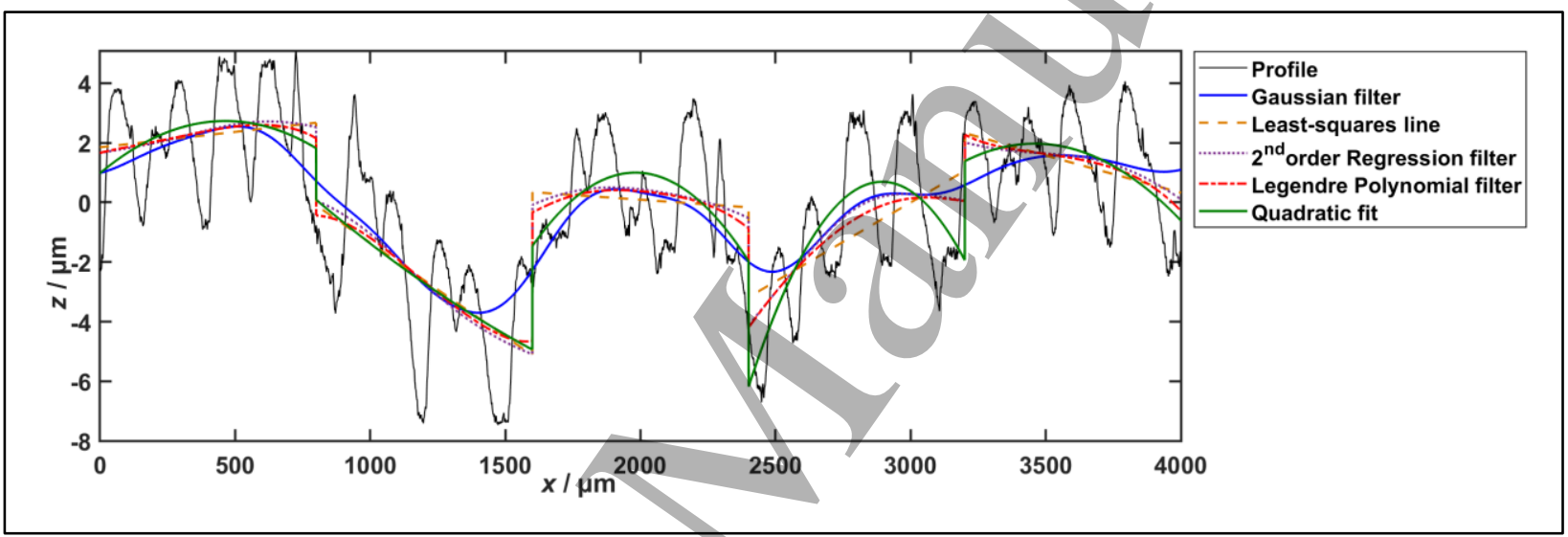

Figure 8. Profile with indicated the Gaussian $w$-filter/over the whole profile of $4 \mathrm{~mm}$ length $\left(5 \cdot \lambda_{c}\right)$, and a linear fit, quadratic fit, the Legendre polynomial filter and the second order Gaussian regression filter, all taken piecewise over separate regions of $0.8 \mathrm{~mm}\left(1 \cdot \lambda_{c}\right)$.

The surface texture $R a$ and $R z$ are taken as the mean value of the 5 cut-off length of the Gaussian filtered profile; according to the definition in ISO 4287 [9]; these are considered as the reference value. Then the mean values of $R a$ and $R z$ of the 5 sub-profiles are taken and averaged. Also the overall rms-difference of the profiles with the Gaussian filtered profile is considered. The results are summarized in table 2, where next to the specimen shown in figure 4 ("Halle 3: rough"), also the results for two other standards of type D1 according to ISO 5436-1 [8] identified as "Halle 2: medium" and "Halle 1: fine" are given. 
Table 2. Parameters $R a$ and $R z$, and the rms difference to the $4 \mathrm{~mm}$ Gaussian filtered profile, given for the unfiltered profile and the mean parameter value for the 5 piecewise profiles filtered with a cut-off length $\lambda_{c}=0.8 \mathrm{~mm}$.

\begin{tabular}{|c|c|c|c|c|c|c|c|c|c|}
\hline & \multicolumn{3}{|c|}{ Halle 1 'fine' } & \multicolumn{3}{|c|}{ Halle 2 'medium' } & \multicolumn{3}{|c|}{ Halle 3 'rough' } \\
\hline Filter & $R a / \mu \mathrm{m}$ & $R z / \mu \mathrm{m}$ & $\begin{array}{l}r m s(\text { diff }) \\
/ \mu \mathrm{m}\end{array}$ & $R a / \mu \mathrm{m}$ & $R z / \mu \mathrm{m}$ & $\begin{array}{l}r m s(\text { diff }) \\
/ \mu \mathrm{m}\end{array}$ & $R a / \mu \mathrm{m}$ & $R z / \mu \mathrm{m}$ & $\begin{array}{l}\text { rms (diff) } \\
/ \mu \mathrm{m}\end{array}$ \\
\hline Unfiltered & 0.206 & 1.213 & 0.142 & 0.787 & 3.58 & 0.551 & 2.236 & 8.67 & 1.68 \\
\hline $\begin{array}{l}\text { Gaussian } \\
\text { filtered }\end{array}$ & 0.147 & 1.157 & - & 0.547 & 2.94 & - & 1.561 & 7.22 & - \\
\hline $\begin{array}{l}0 \text {-order } \\
\text { regression } \\
\text { filter }\end{array}$ & 0.145 & 1.145 & 0.038 & 0.534 & 2.90 & 0.167 & 1.531 & 7.10 & 0.68 \\
\hline $\begin{array}{l}2^{\text {nd }} \text {-order } \\
\text { regression } \\
\text { filter }\end{array}$ & 0.146 & 1.147 & 0.040 & 0.544 & 2.94 & & & 7.15 & 0.68 \\
\hline $\begin{array}{l}\text { Legendre } \\
\text { polynomial }\end{array}$ & 0.146 & 1.147 & 0.037 & 0.550 & 2.95 & 9 & 1.535 & 7.24 & 0.65 \\
\hline Linear fit & 0.151 & 1.175 & 0.047 & 0.586 & 3.16 & 0.957 & 1.621 & 7.73 & 0.74 \\
\hline Quadratic fit & 0.144 & 1.145 & 0.051 & 0.541 & 2.92 & 0.214 & 1.494 & 6.83 & 0.77 \\
\hline
\end{tabular}

Table 2 shows that the zero-order and second order Gaussian regression filter as well as the Legendre polynomial filter show - when averaged over the 5 sections - small but not dramatic differences with the idealized Gaussian filter; average deviations are in the order of $1 \%$ of the measured value. In earlier work a systematic deviation of $-4 \%$ was observed when taking the standard Gaussian filter including endeffects in the piecewise profiles [10]. The similar operation between the filters can be explained from the limited filtering effect of a single cut-off length combined with the least-squares line that has to be subtracted from the profile first anyhow, also for the regression filters. The further operation of these filters is rather weak as in fact all measurement points are in an edge zone (every point is within $\lambda_{c} / 2$ from an edge) but for the regression filters this appears to be well compensated by the additional effect of the least-squares form removal. For the Legendre polynomial filter, little filtering effect remains after removing the least squares line, as can directly be seen in Table 1 as well. A peculiar side-result is that a second-order polynomial (quadratic) fit, that can e.g. be used to remove a spherical of cylindrical form, has already more effect than the other filters. So when a spherical or cylindrical form must be removed, additional filtering makes no sense for profiles like these, as the form removal has already more effect than the regression- or Legendre filtering. Also this can be predicted from Table 1: a second order form removal is defined as $k(0,1,2)=1$ in Table 1 , so its filtering effect will be more than the coefficients listed for $L / \lambda_{c}=1$.

\section{Measurement examples in 2.5-D}

\subsection{Remelted AM-surface}

As a first measurement example in 2.5-D, a part of a remelted additive manufactured surface is taken. It is a piece of maraging steel 300, built on a 3D systems ProX320 laser powder bed fusion machine from 3D Systems, under argon atmosphere. Details can be found in [11]. The measurements are taken with a Mitutoyo CS 3200 formtracer. 300 measurement lines in the $y$-direction are taken with $20 \mu \mathrm{m}$ spacing. Every line has a measurement length of $4 \mathrm{~mm}$ and consists of 2000 measurement points. This 4 x $6 \mathrm{~mm}$ measurement area is taken as unfiltered, filtered with a zero-order areal Gaussian regression filter with an L-nesting index $\left(\lambda_{c}\right)$ of $4 \mathrm{~mm}$ in both directions, and filtered with a 2-D Legendre filter as described in section 2 with the same nesting indices. The results are shown in figure 9. As expected the filtering effect is small for both filter types, and they act very similar on this area. This is also shown in the calculated $S a$ 
parameters according to ISO 25178-2 [12]: The unfiltered topography gives $S a=8.08 \mu \mathrm{m}$, the Gaussian regression filtered topography gives $S a=7.51 \mu \mathrm{m}$ and the Legendre polynomial filtered topography gives $S a=7.52 \mu \mathrm{m}$; the latter values are very close.

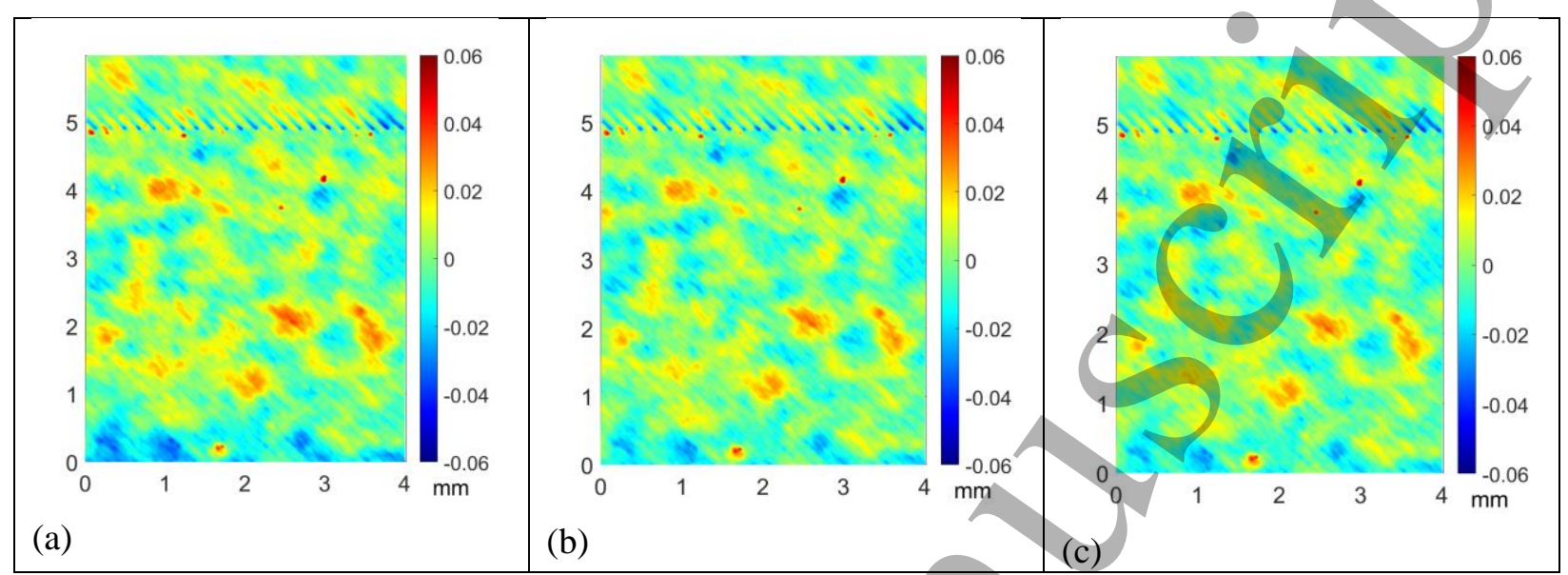

Figure 9. Surface topography a an AM-manufactured and re-melted specimen, unfiltered (a), filtered with a zero-order Gaussian regression filter (b), filtered with a Legendre polynomial filter (c); the L-nesting index was $4 \mathrm{~mm}$ in both directions for both filters.

As in this example there is little filtering effect at all, the similarity between the results does not illustrate much of the differences between the filter types, though it shows the feasibility of the Legendre polynomial filter for this case. The next section provides an example with more prominent and distinguishable filter effects.

\subsection{Hybrid laser-electrochemically machined cavity}

As a second example a hybrid laser-electrochemically machined cavity is taken. Its manufacture by a pulsed laser is described in detail in [13]. The topography of the $993 \mu \mathrm{m} \times 773 \mu \mathrm{m}$ area as presented in figure 10 was obtained using a Sensofar Neox microscope in continuous confocal mode using a 20x objective with a numerical aperture of 0.45 .

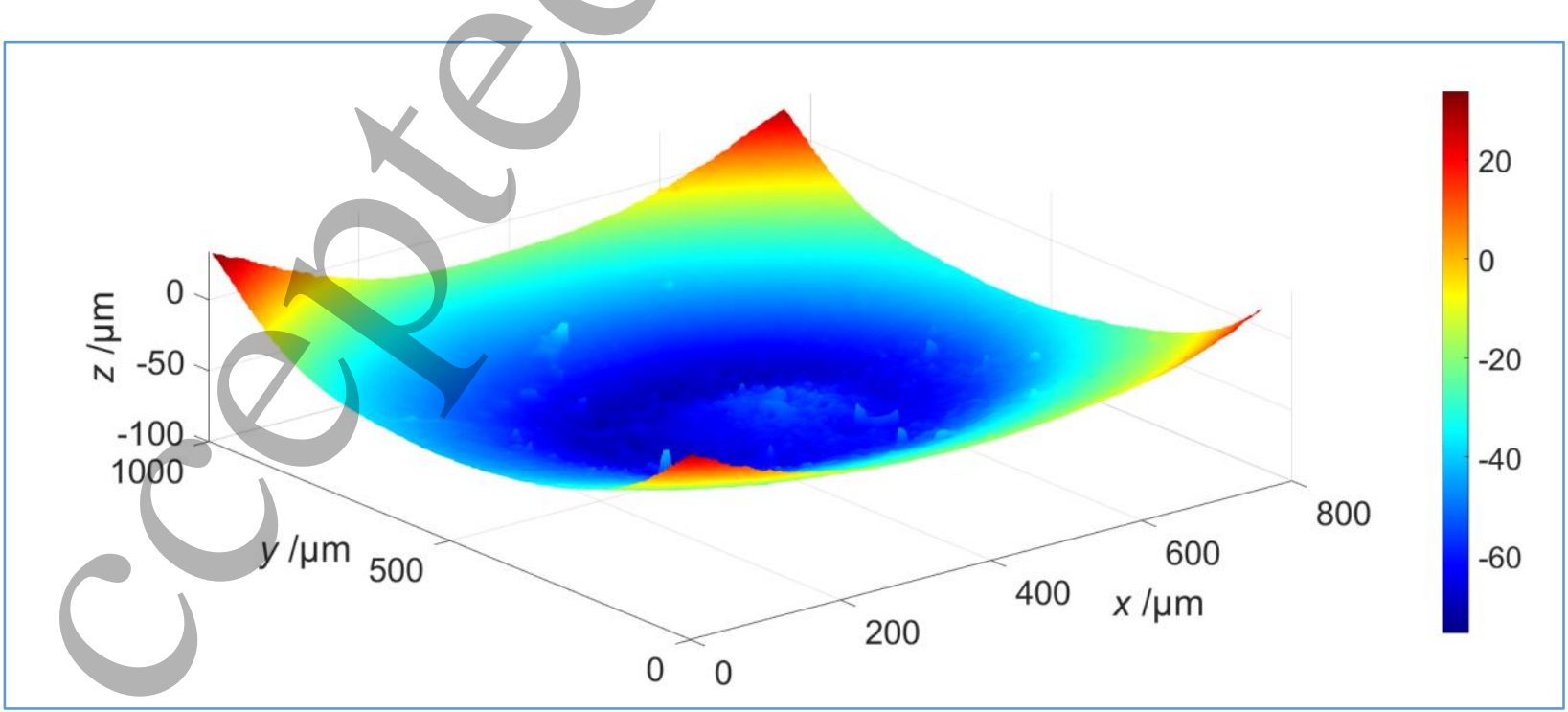

Figure 10. Surface topography of a laser-electrochemically machined cavity 
From this topography, the difference between the Legendre polynomial filtering and a more conventional filtering by a $2^{\text {nd }}$ order Gaussian regression filter is shown by filtering this topography with an L-nesting index $\left(\lambda_{c}\right)$ of $250 \mu \mathrm{m}$. Before the filtering by the $2^{\text {nd }}$ order Gaussian regression filter, the form is removed by a second order 2-D polynomial fit. When applying the Legendre polynomial filter this polynomial fit is part of the filter operation right away. The result for the filtered profiles is shown in figure 10 . The figure shows that some of the form is still remaining in both filtered topographies; this is due to the moderate filter of $\lambda_{c}=250 \mu \mathrm{m}$. The parameter values are $S q=1.34 \mu \mathrm{m}$ and $S q=1.37 \mu \mathrm{m}$ for the Gaussian and the Legendre filtered topographies respectively, but this rather small difference is less relevant than the different filtered topographies obtained. In the $2^{\text {nd }}$ order Gaussian filtered topography some more of the form appears not to be filtered near the edges, despite the local second-order fit that is the characteristic of the $2^{\text {nd }}$ order Gaussian regression filter and the form removal by a $2^{\text {nd }}$ order polynomial fit. Near the center the $2^{\text {nd }}$ order Gaussian regression filter seems to be more efficient in the local form removal; this may be due to the somewhat steeper filter characteristics compared to the standard Gaussian filter. The circular symmetry of the remaining form seems to be better preserved by the Legendre polynomial filter; this may be due to a residual edge effect of the regression filter. The properties of the $2^{\text {nd }}$ order Gaussian regression filter can be improved by fitting a higher order polynomial before filtering, which is to some extent equivalent to applying a Legendre polynomial filter first. This illustrates a gradual transition that may exist between the filter types where it comes to form removal, where the Legendre filter contains the form removal inherently.

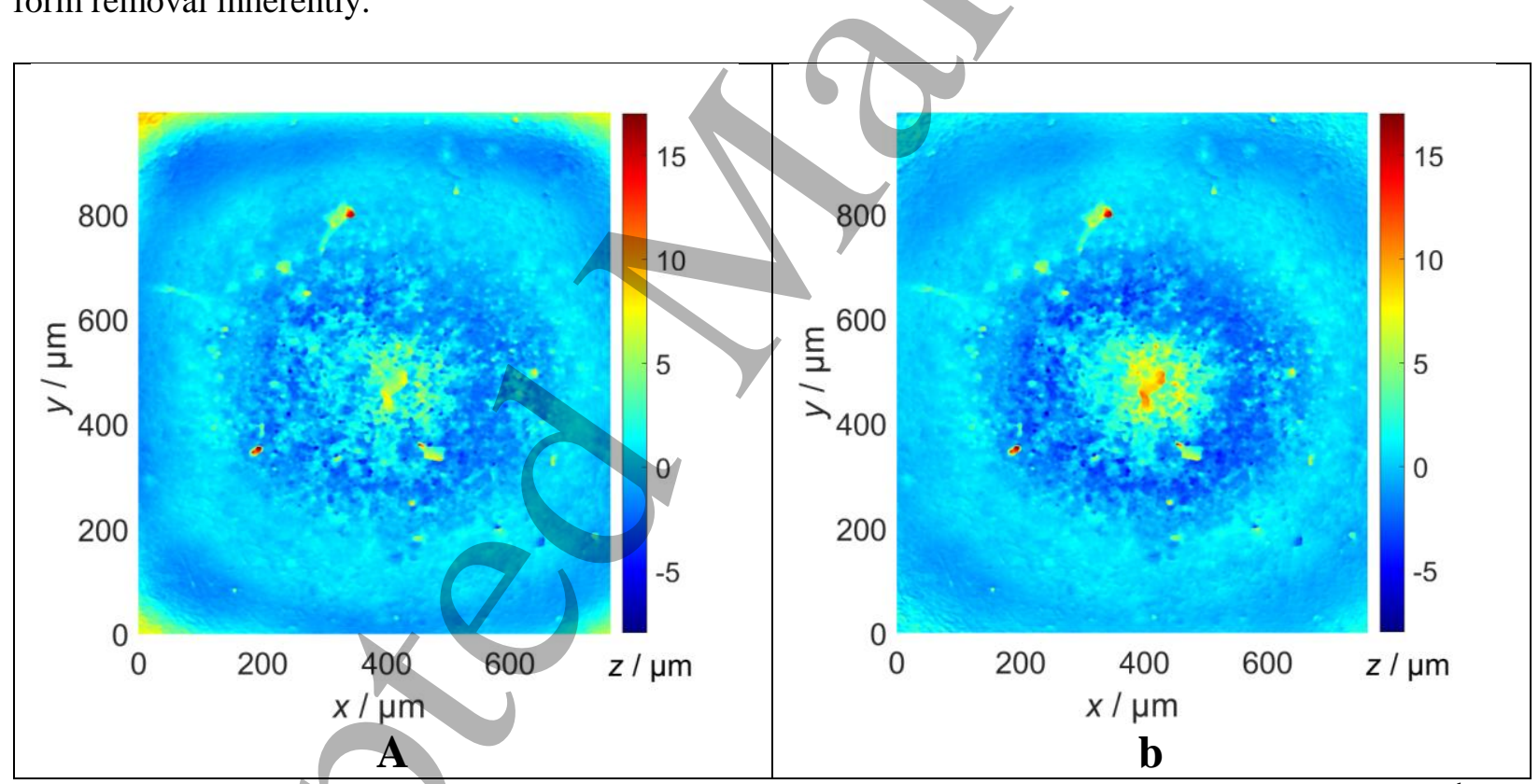

Figure 11 . Filtered surface topography of a laser-electrochemically machined cavity obtained by a $2^{\text {nd }}-$ order Gaussian regression filter (left, a) and by a Legendre polynomial filter (right, b) both with an Lnesting index of $250 \mu \mathrm{m}$.

\section{Numerical considerations}

When continuous Legendre polynomials are considered, which can well be done for more than 200 data points, the coefficients of the Legendre polynomials can be estimated from a standard polynomial leastsquares fit to the data. As shown in table 1 , it is enough to calculate a $5^{\text {th }}, 8^{\text {th }}$ or $11^{\text {th }}$ order polynomial for $L / \lambda_{c}=1, L / \lambda_{c}=3 / 2$ and $L / \lambda_{c}=2$ respectively. It is convenient to scale the $\mathrm{x}$-values in the $[-1,1]$ 
interval. Conversion from the least-squares Taylor-series coefficients to Legendre polynomial coefficients can conveniently be made using a transformation matrix method [14] as following:

A profile can be fitted to both Legendre and standard (Taylor) polynomials represented by coefficients $a_{k}$ and $b_{k}$ respectively up to degree $K$ :

$$
z(x)=\sum_{k=0}^{K} a_{k} P(x, k)=\sum_{k=0}^{K} b_{k} x^{k} .
$$

The continuous Legendre polynomials $P(x, k)$ can be derived from $P(x, 0)=1$ and $P(x, 1)=x$ and the recurrence relationship:

$$
(k+1) \cdot P(x, k+1)=(2 k+1) \cdot x \cdot P(x, k)-k \cdot P(x, k-1) .
$$

For example, for a $5^{\text {th }}$ order polynomial fit this can be written as:

$$
\left(\begin{array}{l}
P(x, 1) \\
P(x, 2) \\
P(x, 3) \\
P(x, 4) \\
P(x, 5) \\
P(x, 6)
\end{array}\right)=\left(\begin{array}{cccccc}
1 & 0 & 0 & 0 & 0 & 0 \\
0 & 1 & 0 & 0 & 0 & 0 \\
-0.5 & 0 & 1.5 & 0 & 0 & 0 \\
0 & -1.5 & 0 & 2.5 & 0 & 0 \\
0.375 & 0 & -3.75 & 0 & 4.375 & 0 \\
0 & 1.875 & 0 & -8.75 & 0 & 7.875
\end{array}\right) \cdot\left(\begin{array}{c}
1 \\
x \\
x^{2} \\
x^{3} \\
x^{4} \\
x^{5}
\end{array}\right)=\boldsymbol{T} \cdot\left(\begin{array}{c}
1 \\
x \\
x^{2} \\
x^{3} \\
x^{4} \\
x^{5}
\end{array}\right)
$$

This means that there is a fixed relation between the coefficient vectors $\overline{a_{k}}$ and $\overline{b_{k}}$ :

$$
\overline{a_{k}}=\boldsymbol{T} \cdot \overline{b_{k}} \text {. }
$$

The matrix $\boldsymbol{T}$ can be expanded to higher orders by adding new rows using the last two rows and (16). When using a $K$-th order least squares polynomial fit, giving the coefficients $\overline{b_{k}}$, the coefficients $\overline{a_{k}}$ can be obtained using the inverse matrix $\boldsymbol{T}^{\mathbf{- 1}}$. The filter function $S(k)$ can be applied to the coefficients $\overline{a_{k}}$ and the modified coefficients of the filtered function are found by applying the transform $\boldsymbol{T}$ again. This gives for the filter function $w(x)$ :

$$
w_{L}(x)=\sum_{k=0}^{K} \overline{b_{f k}} \cdot x^{k}
$$

Where $b_{f k}$ are the filtered polynomial coefficients given by:

$$
\overline{b_{f k}}=\boldsymbol{T} \cdot\left(S(k) \cdot\left(\boldsymbol{T}^{-1} \cdot b_{k}\right)\right)
$$

This calculation consists of a least-squares polynomial fit, that is standard available in most software packages, and a few matrix- and vector operations. This works straightforward for a profile, but is less straightforward for an areal topography.

For a discrete function $z(x)$ the normalized discrete Legendre polynomial functions can be calculated using (9). However if only a few, $K$, polynomials are needed it is computationally more efficient to use the recurrence relation given by Neuman and Schonbach [15]:

$\quad(k+1) \cdot(N-k) \cdot P(x, k+1, N)=(2 k+1) \cdot(N-2 x) \cdot P(x, k, N)-k \cdot(N+k+1) \cdot P(x, k-1, N)$,
for $k=1,2 . .(K-1), K<N$ with the initial values $P(x, 0, N)=1$ and $P(x, 1, N)=(N-2 x) / N$

These polynomials can be normalized using: 


$$
p_{n}(x, k, N)=\frac{P(x, k, N)}{\sqrt{\sum_{x=0}^{N-1} P^{2}(x, k, N)}},
$$

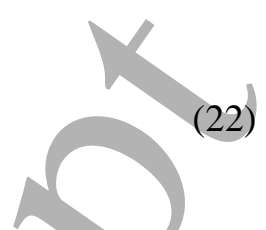
giving the same result as (9). The filtering is carried out using (8) and (11), and (14) for the 2.5-D case. This method proved to be most computationally efficient, and polynomials up to $k=50$ can be computed numerically stable in this way. The discrete Legendre polynomials have the additional advantage that they keep their orthogonality, also for smaller amounts of data. Filtering a 2.5-D topography consisting of $1000 \times 1000$ measured points, using $L / \lambda_{c}=1.5$, took $0.27 \mathrm{~s}$ using Matlab R2019b on a Dell Latitude 7490 with 16 GB RAM. The code is not yet compiled so this calculation time can well be reduced if needed. As a comparison, an FFT-based Gaussian filter applied to the same surface took $0.08 \mathrm{~s}$, while a Gaussian regression filter based on convolution took 3 seconds, where it must be noted that algorithms for fast Gaussian filtering based on convolution have been developed that take little more time than the FFTbased algorithm $[16,17]$. This means that the Legendre filter may take somewhat more, but no excessive calculation time compared to existing filter algorithms.

\section{Conclusions and outlook}

A filtering method is designed based on discrete Legendre polynomials that can be used to separate form and roughness form surface profiles and areal topographies. The filter has been designed in such a way that it well approaches the properties of a Gaussian filter, especially in the center of the measured region. In the cases studied there is little difference with the zero- and second order Gaussian regression filters; both in the obtained topography and the calculated profile- and areal parameters. The principal advantage is that the form removal is an integral part of the filtering and does not need to be carried out separately. Also the defined form and reference plane are principally unchanged after application of this filter. Because the Legendre polynomials are defined on a specified range and do not assume periodicity, there are no edge effects. Compared to the Gaussian regression filters the filtering becomes gradually weaker outside the center of the profile/area, while for the Gaussian Regression filters the filtering is constant over the profile/area, but becomes weaker at the edge region within half of the L-nesting index. For the default case according to ISO 25178-3 [3], where the nesting index is equal to the surface size, this filter is a viable alternative because of the principal advantages mentioned. For an L-filter with a smaller Lnesting index than the measurement range also a quadratic or higher-order polynomial form removal can be integrated where the form remains independent of further filtering. This can be an advantage when applying the Legendre polynomial filter to topographies of workpieces with complicated forms.

\section{Acknowledgements}

The following co-workers from the KU Leuven are acknowledged: Jitka Metelkova for making the maraging steel measurements available and Maxim Vanrusselt and Krishna Saxena for providing the cavity measurement.

\section{References}

[1] David J Whitehouse 1993 Handbook of Surface Metrology, CRC Press

[2] Brinkman S Bodschwinna H 2003 Advanced Gaussian Filters, in: Advanced Techniques for Assessment Surface Topography: Development of a Basis for 3D Surface Texture Standards "Surfstand", 63, Elsevier.

[3] ISO 25178-3 2012 Geometric product specifications(GPS) - Surface texture: Areal— Part 3: Specification operators (Geneva: Int. Org. for Standardization) 
[4] Haitjema H 2020 Straightness, flatness and cylindricity characterization using discrete Legendre polynomials CIRP Annals 69 (1) 457

[5] ISO 16610-21 2011 Geometric product specification (GPS) - filtration - Part 21: Linear profile filters: Gaussian filters (Geneva: Int. Org. for Standardization) .

[6] Morrison, N, 1969 Introduction to Sequential Smoothing and Prediction, McGraw Hill, New York

[7] ISO 16610-31 2016 Geometric product specification (GPS) - filtration - Part 31: Robust profile filters: Gaussian regression filters (Geneva: Int. Org. for Standardization).

[8] ISO 5436-1 2000 Geometrical Product Specification (GPS) - surface texture: Profile method; Measurement standards - Part 1: Material measures (Geneva: Int. Org. for Standardization)

[9] ISO 42871997 Geometrical Product Specification (GPS) - surface texture: Profile method; Terms, definitions and surface texture parameters (Geneva: Int. Org. for Standardization)

[10] Leach R Haitjema H 2010 Bandwidth characteristics and comparisons of surface texture measuring instruments Measurement Science and Technology 21 (3) 032001

[11] Metelkova J De Formanoir C Haitjema H Witvrouw A Pfleging W Van Hooreweder B 2019 Elevated edges of metal parts produced by laser powder bed fusion: characterization and post-process correction. Proceedings of Joint Special Interest Group Euspen/ASPE, Nantes, France

[12] ISO 25178-3 2012 Geometric product specifications(GPS) - Surface texture: Areal— Part 2: Terms, definitions and surface texture parameters (Geneva: Int. Org. for Standardization)

[13] Saxena K K Vanrusselt M Qian J Reynaerts D Haitjema H 2020 Characterization of hybrid laserelectrochemically machined cavities by areal surface roughness parameters Proceedings of the 20th International Conference of the European Society for Precision Engineering and Nanotechnology, EUSPEN 2020171

[14] Sparis P D Mouroutsos S G 1985 The operational matrix of polynomial series transformation, International Journal of Systems Science 16 (9) 1173

[15] Neuman C P Schonbach D I 1974 Discrete (Legendre) orthogonal polynomials - a survey International Journal for Numerical Methods in Engineering 8 (4) 743

[16] Krystek M 1996 A fast Gauss filtering algorithm for roughness measurements. Precision Engineering 19198

[17] Kong M Guan Q, Zhao J 2014 A fast algorithm of robust Gaussian regression filter Zhongguo Jixie Gongcheng/China Mechanical Engineering 25 (6) 776 


\section{Appendix 1: Matlab code for 2.5-D Legendre filtering}

The function for performing the Legendre filtering in 2.5-D:

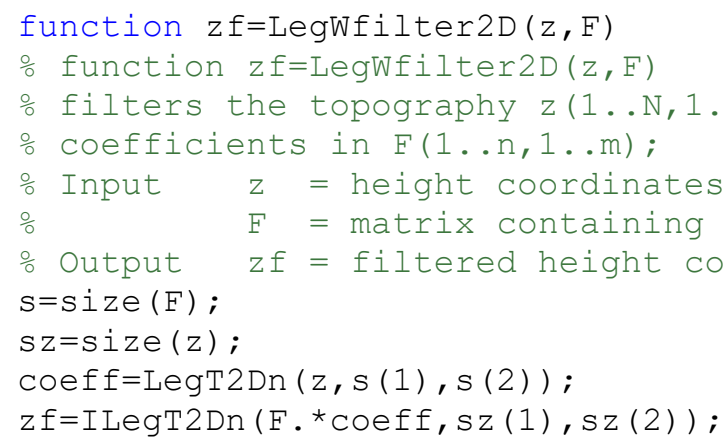

The 2.5-D Legendre transform:

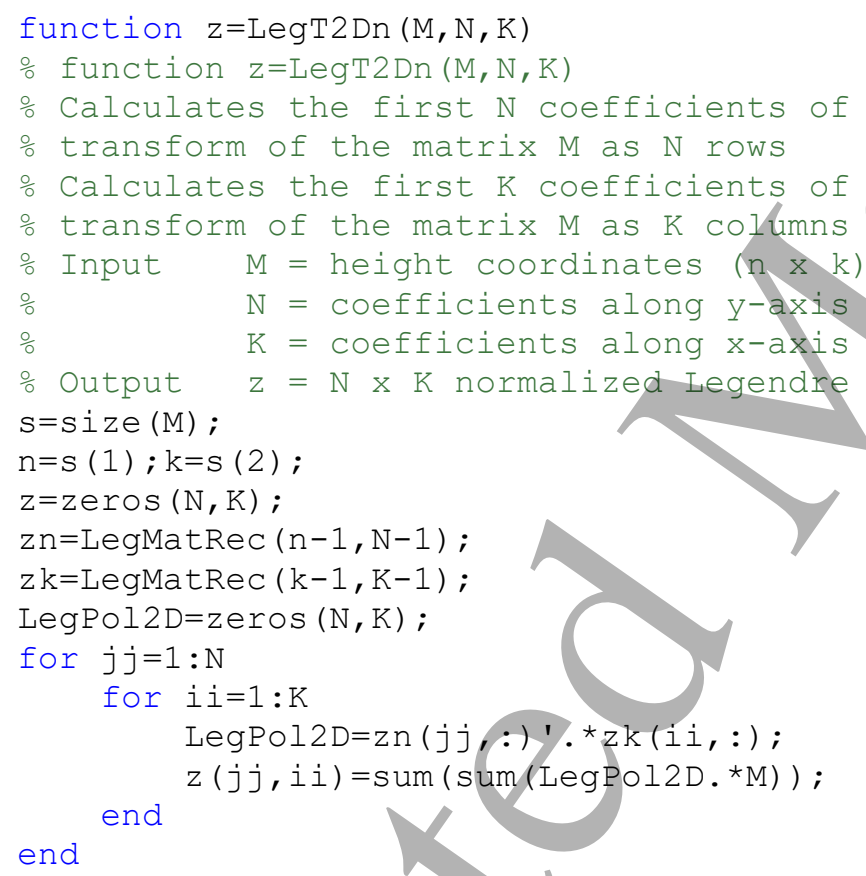

The 2.5-D inverse Legendre transform

function $\mathrm{z}=\operatorname{ILegT2Dn}(\mathrm{M}, \mathrm{N}, \mathrm{K})$

을

\% Calculates the first $N$ coefficients of the 2-D discrete Inverse Legendre

o transform of the matrix $M$ as $N$ rows

o Calculates the first K coefficients of the 2-D discrete Inverse Legendre

o transform of the matrix $M$ as $K$ columns

Input $M=$ Legendre coefficients ( $\mathrm{n} x \mathrm{k}$ ) along equidistant $y$ and $\mathrm{x}-\mathrm{axis}$

( $\mathrm{N}=$ coordinates along $\mathrm{y}$-axis (first coordinate) $\mathrm{n}<=\mathrm{N}$

ㅇ $\mathrm{K}=$ coordinates along $\mathrm{x}$-axis (second coordinate) $\mathrm{k}<=\mathrm{K}$

output $\mathrm{z}=\mathrm{N}$ x $\mathrm{K}$ z coordinates

$\mathrm{s}=\mathrm{size}(\mathrm{M})$;

$\mathrm{n}=\mathrm{s}(1) ; \mathrm{k}=\mathrm{s}(2)$; 


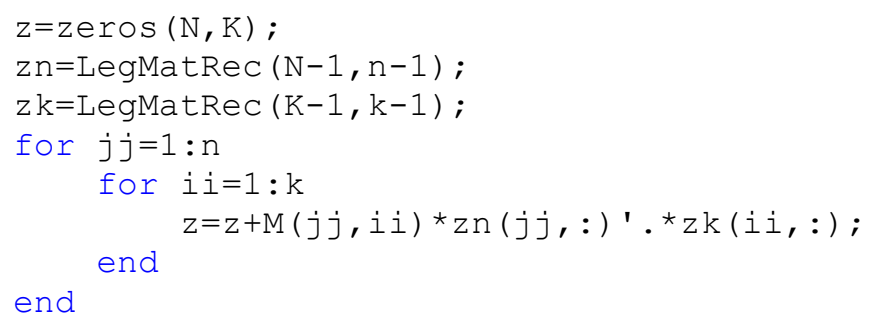

The Legendre matrix given in (9) using the recurrence relationship in (21) and the normalization in (22)

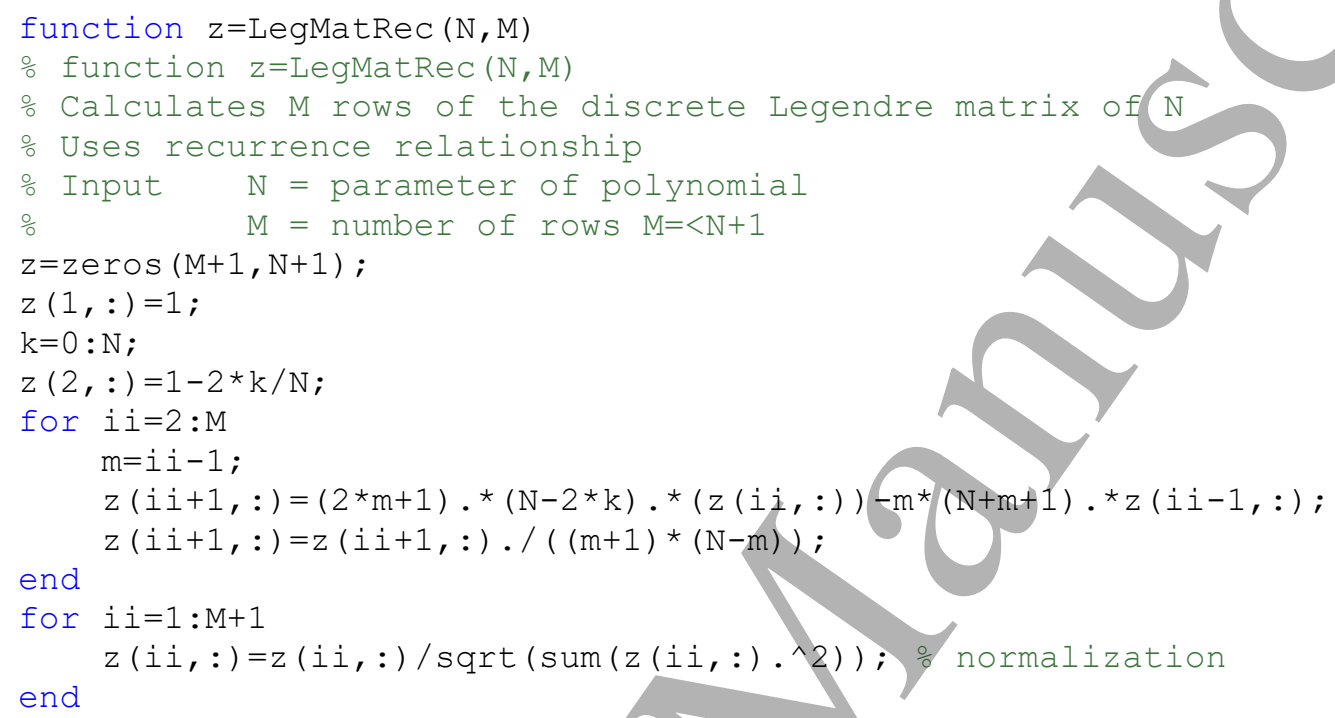

